\title{
On-line Parameter Adaptation for a Momentum Control in the Post-Grasping of a Tumbling Target with Model Uncertainty
}

\author{
Satoko Abiko and Gerd Hirzinger \\ Institute of Robotics and Mechatronics \\ German Aerospace Center (DLR) \\ 82334, Weßling, Germany \\ satoko.abiko@dlr.de
}

\begin{abstract}
This paper addresses an on-line parameter adaptation for a momentum accumulation control of a space robot in the post-grasping of a tumbling target whose dynamic parameters are unknown a priori. The model inaccuracies in the target lead to an unexpected tumbling motion after grasping a target. It is desired to transfer the entire angular momentum to the reaction wheels as quickly as possible while stabilization trajectory of the robot-arm is tracked to avoid self-collision in the chaser-robot.

Firstly, we derive a momentum control method from the angular momentum equation to accumulate the entire angular momentum into the reaction wheels. The parameter inaccuracies degrade the control performance. Then, an online adaptation law by using coupling force and momentum is proposed. A numerical simulation is carried out to verify the operational performance of the proposed method in the presence of model uncertainty.
\end{abstract}

Index Terms-On-Line Parameter Adaptation, Momentum Control, Free-Floating Space Robot, Model Uncertainty

\section{INTRODUCTION}

The capture of a free-floating target on orbit is a highpriority task in the field of on-orbit space robotics since various autonomous tasks, such as inspection, assembly and maintenance on orbit, can be achieved once practical techniques for the capturing operation are established.

This paper addresses the task of grasping a tumbling target by means of a free-floating space robot. The target is assumed to be initially tumbling in some given arbitrary free motion. Following an operational strategy by which the impact between the robot end-effector and the target is minimized at the grasp, the subsequent stabilization motion is analyzed here for the case of uncertainty in the target dynamic model. This leads to an unexpected tumbling motion of the entire system, consisting of the chaser-robot and the target, where a given nominal stabilization trajectory has to be tracked, while accounting for the parameter uncertainty. In order to stabilize the chaser-robot with a target, therefore, a prompt momentum accumulation is required while avoiding self-collisions in the chaser-robot.

In this paper, we propose an on-line parameter adaptation method to account for unknown total angular momentum and unknown dynamic parameters in the post-grasping phase of the capturing operation. The proposed method is verified

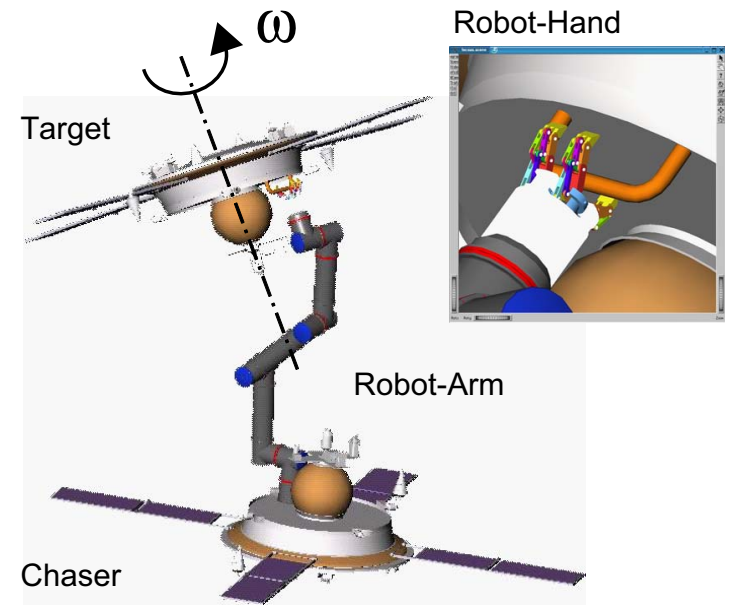

Fig. 1: Chaser-robot and target scenario

by a numerical simulation for a realistic three-dimensional scenario (see Fig. 1).

The paper is organized as follows. Section II shows brief literature survey for previous researches. Section III explains the mission scenario and assumptions. Section IV describes dynamic models of a space robot. Section V discusses a control for post-grasping of a tumbling target. Section VI proposes an on-line parameter adaptation algorithm. Section VII illustrates simulation results with a three-dimensional model. The conclusions are summarized in Section VIII.

\section{BRIEF LITERATURE SURVEY}

The space mission to capture a tumbling target by means of a chaser-robot may be divided into four main phases:

(1) approaching and following the target motion (Pre-grasping),

(2) capturing the target with the robot-arm (Contact),

(3) damping out the motion of the target relative to the chaser-satellite (Post-grasping),

(4) stabilizing the tumbling motion of the compound system (Compound stabilization).

Phases (3) and (4) are usually categorized together as a postgrasping phase in the previous researches. The authors divide 
them into phase (3) and (4) to comprehend two inherent issues easily. Phase (3) is a trajectory control task for the manipulator arm to avoid self-collision. Phase (4) is the task to deal with angular momentum of the whole system. This paper addresses both together in the case when the dynamic properties of the target are not known in advance.

To cope with the model uncertainty, $\mathrm{Xu}$ and $\mathrm{Gu}$ proposed adaptive control schemes for space robots in both joint space and operational space [1], [2]. These approaches, however, focus on the tracking error issues on the assumption that the dynamic parameter distribution in the entire system is unknown and the total momentum is known. They have not discussed the treatment of the unknown total angular momentum.

In [3], both damping out of the chaser-target relative motion and the following compound stabilization are dealt with simultaneously by using the principle of the conservation of momentum. The main focus in [3] is on minimizing the attitude change of the base-satellite before and after the grasping of a target. This approach, however, has assumed that the angular momentum of the target is known in advance, which is very difficult in practice due to the dynamic parameter inaccuracies in the target. Eventually the completion of the capturing operation is considered when the angular momentum on the target is transfered into the reaction wheels on the chaser-satellite. In addition, it is also hard to find an arbitrary trajectory in the approaching phase and the post-grasping phase in [3]. This severe trajectory limitation may lead to a failure of the reaching to the grasping point on the target in the approaching phase and it may lead to the self-collision in the chaser-robot in the post-grasping phase.

To avoid those crucial situations, the motion planner gives a certain trajectory to the robot arm in our strategy. The remaining issue is the treatment of the unknown angular momentum for the entire system after grasping the target with model uncertainty. Then, this paper discusses an online parameter adaptation algorithm for fast accumulation of an unknown entire angular momentum into the reaction wheels while the stabilization trajectory is tracked.

With regards to phase (2), some previous researches have analyzed the impact between the target and the manipulator [4]-[6]. These studies, however, do not consider the capturing of the target, nor the model uncertainty in its dynamics.

\section{MISSION SCENARIO AND ASSUMPTIONS}

In our envisioned scenario, it is assumed that an inverse kinematics algorithm provides the ideal robot trajectory to align the end-effector velocity with that of the grasping point on the target, such that the impact at the grasp is minimal. This may be achieved by means of an ideal visual path tracker. Subsequently, a motion planner provides a stabilization trajectory to bring the relative motion between the robot and the target to zero, while ensuring feasibility of the task.

In the above task, we assume that:

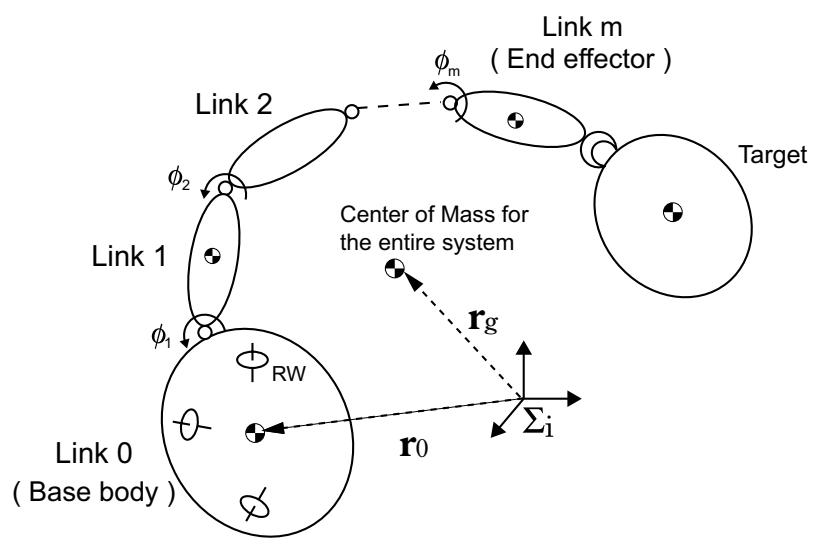

Fig. 2: Model of a chaser-robot grasping a target

$a_{1}$ ) the target is initially tumbling and its dynamic parameters and angular momentum are not known in advance;

$a_{2}$ ) the target motion rate is observed by an ideal visual tracker.

$a_{3}$ ) the total angular momentum of the chaser-robot before grasping a target is known, here it is supposed to be zero;

$a_{4}$ ) no external forces are induced into the entire system (a chaser-robot and a target). The reaction wheels are used for angular momentum management. No gas-jet thrusters are used in the chaser-satellite;

$a_{5}$ ) the post-grasping operation is successfully completed when the total angular momentum is transfered into the reaction wheels on the chaser-satellite;

$a_{6}$ ) the stabilization trajectory of the robot-arm is designed in advance for the avoidance of the self-collision and for the joint limitation. Each joint follows the given trajectory by the velocity-based closed-looped servo controller, then here the velocity is considered as an input command;

$a_{7}$ ) each joint angle and motion ratio are measurable;

$a_{8}$ ) the angular velocity of the base-satellite are measurable;

$a_{9}$ ) the joint torques are measurable or the reaction forces due to the unknown target is measurable with the force/torque sensor attached on the end-effector.

\section{MODELING AND EQUATIONS OF MOTION}

\section{A. Equations of Motion}

An articulated robot arm mounted on a base-satellite is regarded as a multi-body system. The equation of motion of the system is generally expressed in the following form [1]:

$$
\begin{aligned}
{\left[\begin{array}{ccc}
w \boldsymbol{E} & w \widehat{\boldsymbol{r}}_{0 g}^{T} & \boldsymbol{J}_{T g} \\
w \widehat{\boldsymbol{r}}_{0 g} & \boldsymbol{H}_{\omega} & \boldsymbol{H}_{\omega \phi} \\
\boldsymbol{J}_{t g}^{T} & \boldsymbol{H}_{\omega \phi}^{T} & \boldsymbol{H}_{\phi}
\end{array}\right] } & {\left[\begin{array}{c}
\dot{\boldsymbol{v}}_{b} \\
\dot{\boldsymbol{\omega}}_{b} \\
\ddot{\boldsymbol{\phi}}
\end{array}\right]+\left[\begin{array}{c}
\boldsymbol{c}_{b_{v}} \\
\boldsymbol{c}_{b_{\omega}} \\
\boldsymbol{c}_{\phi}
\end{array}\right]=} \\
& {\left[\begin{array}{c}
\mathcal{F}_{b} \\
\mathcal{T}_{b} \\
\boldsymbol{\tau}
\end{array}\right]+\left[\begin{array}{c}
\boldsymbol{J}_{b_{v}}^{T} \\
\boldsymbol{J}_{b_{\omega}}^{T} \\
\boldsymbol{J}_{\phi}^{T}
\end{array}\right] \mathcal{F}_{h} }
\end{aligned}
$$

where the generalized coordinates are defined to be the linear and angular velocity of the base-satellite, $\dot{\boldsymbol{x}}_{b}=$ 
$\left(\boldsymbol{v}_{b}^{T}, \boldsymbol{\omega}_{b}^{T}\right)^{T} \in R^{6 \times 1}$, and the motion rate of the active joints, $\dot{\phi} \in R^{n \times 1}$. This formulation is not limited to a single, seriallink manipulator arm. In this paper, we assume one serial manipulator system with $m$ Degrees-Of-Freedom (DOF) and $l$ DOF reaction wheels (RW) are mounted on the basesatellite as shown in Fig. 2, then the motion rate of the active joints can be separated into $\dot{\boldsymbol{\phi}}=\left(\dot{\boldsymbol{\phi}}_{m}^{T}, \dot{\boldsymbol{\phi}}_{r}^{T}\right)^{T}$ where $\dot{\boldsymbol{\phi}}_{m}$ is for the robot-arm and $\dot{\phi}_{r}$ is for the reaction wheels. Then, total DOF of the actuated joints are the sum of the DOF of the robot-arm and that of the reaction wheels, $n=m+l$. The symbols used in eq. (1) are defined as follows:

$$
\begin{gathered}
\boldsymbol{H}_{b}=\left[\begin{array}{cc}
w \boldsymbol{E} & w \widehat{\boldsymbol{r}}_{0 g}^{T} \\
w \widehat{\boldsymbol{r}}_{0 g} & \boldsymbol{H}_{\omega}
\end{array}\right] \in R^{6 \times 6} \\
\boldsymbol{H}_{b c}=\left[\begin{array}{ll}
\boldsymbol{H}_{b m} & \boldsymbol{H}_{b r}
\end{array}\right]=\left[\begin{array}{c}
\boldsymbol{J}_{T g} \\
\boldsymbol{H}_{\omega \phi}
\end{array}\right] \in R^{6 \times n}
\end{gathered}
$$

where $\boldsymbol{H}_{b}\left(\boldsymbol{x}_{b}, \boldsymbol{\phi}\right)$ and $\boldsymbol{H}_{b c}\left(\boldsymbol{x}_{b}, \boldsymbol{\phi}\right)$ denote the base inertia matrix and the augmented coupling inertia matrix, respectively. $\boldsymbol{H}_{b c}$ consists of the coupling inertia matrix between the robot-arm and the base $\boldsymbol{H}_{b m}$, and the one between the reaction wheels and the base $\boldsymbol{H}_{b r} . w$ is the total mass of the system, $\boldsymbol{E} \in R^{3 \times 3}$ is the identity matrix and $\boldsymbol{r}_{0 g}=\boldsymbol{r}_{g}-\boldsymbol{r}_{0}$ stands for the vector from the centroid of the base to the total mass center of the system (Fig. 2). The operators $\{\cdot\}^{T}$ and $\widehat{\{\cdot\}}$ denote a matrix transpose and a skew symmetric matrix, respectively.

$$
\begin{aligned}
& \boldsymbol{H}_{\phi} \in R^{n \times n} \quad: \quad \text { inertia matrix of the active joints. } \\
& \boldsymbol{c}_{b} \in R^{6 \times 1} \quad \text { : non-linear velocity dependent term } \\
& \text { of the base. } \\
& \boldsymbol{c}_{\phi} \in R^{n \times 1} \quad: \quad \text { non-linear velocity dependent term } \\
& \text { of the active joints. } \\
& \mathcal{F}_{h} \in R^{6 \times 1} \quad: \quad \text { force and moment exerted on the } \\
& \text { end-effector. } \\
& \mathcal{F}_{b} \in R^{6 \times 1} \quad: \quad \text { force and moment exerted on the } \\
& \text { base. } \\
& \tau \in R^{n \times 1}: \text { torque on the active joints. }
\end{aligned}
$$

Notations for the sub-matrices which are not shown here can be found in [1].

\section{B. Linear and Angular Momentum Equations}

The dynamic equation in relation to the motion of the chaser-base is extracted from the upper part of eq. (1) with no external force on the end-effector and the base-satellite:

$$
\mathcal{F}=\left[\begin{array}{c}
\mathcal{F}_{b} \\
\mathcal{T}_{b}
\end{array}\right]=\boldsymbol{H}_{b} \ddot{\boldsymbol{x}}_{b}+\boldsymbol{H}_{b c} \ddot{\boldsymbol{\phi}}+\boldsymbol{c}_{b},
$$

where $\boldsymbol{c}_{b}=\left(\boldsymbol{c}_{b_{v}}^{T}, \boldsymbol{c}_{b_{\omega}}^{T}\right)^{T}$ and $\boldsymbol{H}_{b c}=\left[\begin{array}{ll}\boldsymbol{H}_{b m} & \boldsymbol{H}_{b r}\end{array}\right]$. The integral of (2) provides the momentum equations of the system around the center of mass of the base, by which the motion of the space robot is governed:

$$
\mathcal{L}=\left[\begin{array}{c}
\boldsymbol{P}_{b} \\
\boldsymbol{L}_{b}
\end{array}\right]=\boldsymbol{H}_{b} \dot{\boldsymbol{x}}_{b}+\boldsymbol{H}_{b c} \dot{\boldsymbol{\phi}}
$$

where $\mathcal{L}=\left(\boldsymbol{P}_{b}^{T}, \boldsymbol{L}_{b}^{T}\right)^{T} \in R^{6 \times 1}$ is linear and angular momentum of the entire system.

\section{Angular Momentum Decomposition}

In grasping a tumbling target, a significant feature is the change of the entire angular momentum. Eliminating the linear velocity of the base from the upper part of (3), one can obtain the following angular momentum equation expressed by the angular velocity of the base $\boldsymbol{\omega}_{b}$ and the motion rate of the active joints $\dot{\phi}=\left(\dot{\phi}_{m}^{T}, \dot{\phi}_{r}^{T}\right)^{T}$.

$$
{ }^{A} \boldsymbol{H}_{\omega} \boldsymbol{\omega}_{b}+{ }^{A} \boldsymbol{H}_{\omega \phi} \dot{\boldsymbol{\phi}}_{m}+{ }^{A} \boldsymbol{H}_{\omega r} \dot{\boldsymbol{\phi}}_{r}={ }^{A} \boldsymbol{L}_{g},
$$

where

$$
\begin{gathered}
{ }^{A} \boldsymbol{H}_{\omega}=\boldsymbol{H}_{\omega}-\frac{1}{w} \widehat{\boldsymbol{r}}_{0 g} \widehat{\boldsymbol{r}}_{0 g}^{T}, \quad\left[{ }^{A} \boldsymbol{H}_{\omega \phi}{ }^{A} \boldsymbol{H}_{\omega r}\right]=\boldsymbol{H}_{\omega \phi}-\widehat{r}_{0 g} \boldsymbol{J}_{T g} \\
{ }^{A} \boldsymbol{L}_{g}=\boldsymbol{L}_{b}-\widehat{\boldsymbol{r}}_{0 g} \boldsymbol{P}_{b} .
\end{gathered}
$$

Equation (4) represents the entire angular momentum around the center of mass of the whole system, which is conserved when no external forces are applied to the system. Eq. (4) gives us some insight into the management of the entire angular momentum, which is expressed in the following section.

\section{Post-GRASPING MOMENTUM CONTROL}

This section discusses the method to accumulate the entire angular momentum as quickly as possible in the presence of dynamic parameter inaccuracies. In the grasping of a tumbling target, whose dynamic parameters are unknown, an unexpected tumbling motion for the whole system can be observed. Firstly, the momentum accumulation control in the case without any model error is proposed. Then, we analyze the operational efficiency of the momentum control law in the case with the dynamic parameter errors. For simplicity, it is assumed that the dynamic parameters of the end-effector are changed due to the grasped target.

\section{A. Momentum Accumulation Control}

After the chaser-robot grasps a tumbling target, the whole dynamic model will change due to the target dynamic properties, especially the entire angular momentum. We propose a control method to treat the angular momentum while the robot-arm follows a stabilization trajectory, which is generated to avoid internal collision in the joint space. The motion of the robot arm, $\dot{\phi}_{m}$, is given as a decaying trajectory, which finally converge to the zero motion ratio with a proper optimal trajectory planning [7]. While the robot-arm is operated by the given velocity profile, the motion rate of the reaction wheels is actuated by the following control law:

$$
\dot{\boldsymbol{\phi}}_{r}={ }^{A} \boldsymbol{H}_{\omega r}^{-1}\left({ }^{A} \boldsymbol{H}_{\omega} \boldsymbol{\omega}_{b}+{ }^{A} \boldsymbol{H}_{\omega \phi} \dot{\boldsymbol{\phi}}_{m}+{ }^{A} \boldsymbol{H}_{\omega r} \dot{\boldsymbol{\phi}}_{r}\right),
$$

where $\boldsymbol{\omega}_{b}, \dot{\phi}_{m}$ and $\dot{\phi}_{r}$ are assumed to be measurable. The above control law gives us the following condition:

$$
{ }^{A} \boldsymbol{H}_{\omega} \boldsymbol{\omega}_{b}+{ }^{A} \boldsymbol{H}_{\omega \phi} \dot{\boldsymbol{\phi}}_{m}=\mathbf{0} .
$$

Consequently, because of the decaying velocity trajectory of the robot arm, the angular velocity of the base $\omega_{b}$ converges 
to zero and the whole angular momentum is accumulated into the reaction wheels. However, the above control law can be achieved efficiently when the dynamic parameters in the entire system, including the target inertial properties, are well-known.

\section{B. The influence of the parameter uncertainty}

Let us discuss the case when the dynamic parameter errors exist in the control model. In the presence of parameter inaccuracies, the control law for the reaction wheels is modified to the following expression including model errors:

$$
\dot{\boldsymbol{\phi}}_{r}={ }^{A} \overline{\boldsymbol{H}}_{\omega r}^{-1}\left({ }^{A} \overline{\boldsymbol{H}}_{\omega} \boldsymbol{\omega}_{b}+{ }^{A} \overline{\boldsymbol{H}}_{\omega \phi} \dot{\boldsymbol{\phi}}_{m}+{ }^{A} \overline{\boldsymbol{H}}_{\omega r} \dot{\boldsymbol{\phi}}_{r}\right)
$$

where $\overline{\{\cdot\}}$ denotes the matrix including the dynamic parameter errors. The error matrix between the real plant and the corresponding model can be expressed as:

$\widetilde{\boldsymbol{H}}_{\omega}=\overline{\boldsymbol{H}}_{\omega}-\boldsymbol{H}_{\omega}, \widetilde{\boldsymbol{H}}_{\omega \phi}=\overline{\boldsymbol{H}}_{\omega \phi}-\boldsymbol{H}_{\omega \phi}, \widetilde{\boldsymbol{H}}_{\omega r}=\overline{\boldsymbol{H}}_{\omega r}-\boldsymbol{H}_{\omega r}$,

where $\widetilde{\{\cdot\}}$ denotes the error matrix. The control law (7) achieves the following closed-loop system:

$$
\boldsymbol{H}_{\omega} \boldsymbol{\omega}_{b}+\boldsymbol{H}_{\omega \phi} \dot{\boldsymbol{\phi}}_{m}=\widetilde{\boldsymbol{H}}_{\omega} \boldsymbol{\omega}_{b}+\widetilde{\boldsymbol{H}}_{\omega \phi} \dot{\boldsymbol{\phi}}_{m}
$$

Equation (8) implies that in spite of the parameter inaccuracies, the decaying velocity trajectory in the robot-arm provides us with the convergence of $\boldsymbol{\omega}_{b}$ to zero and the transfer of the entire angular momentum into the reaction wheels. However, eq. (8) explains that while the robotarm is moving, the whole angular momentum can not be transfered into the reaction wheels completely due to the dynamic inaccuracies, and then an unexpected tumbling motion maintains unless the robot-arm is stopped. Note that the control response of the reaction wheels is adjusted properly. However, the model inaccuracies degrade the momentum accumulation performance of the control (7). To cope with this issue, on-line parameter adaptation is useful.

\section{ON-LINE PARAMETER ADAPTATION ALGORITHM}

In this section, we propose an on-line parameter adaptation method to control the angular momentum of the entire system properly against the model uncertainties. Firstly, we explain the linearity in the dynamic parameters, which is a significant property to derive the adaptation algorithm to be proposed. The proposed adaptation algorithm requires measurement values of the coupling force between the basesatellite and the robot-arm, which are used for so-called prediction error for parameter identification [8]. The stability of the proposed adaptation algorithm is proved by means of the Lyapunov direct method and its parameter convergence is analyzed.

\section{A. Linearity in the dynamic parameters}

To deduce an adaptation law, let us discuss the linearity in terms of the dynamic parameters characterizing a space robot. It is well-known that the dynamic model for an articulated multi-body system can be linearized with respect to a proper set of the dynamic parameters [9] and a space robot is modeled in the same formulation. In analogy with
[9], the dynamic model of space robots (2) and (3) can be expressed in the linear parameterization forms:

$$
\begin{aligned}
\mathcal{F} & =\boldsymbol{y} \boldsymbol{a}=\boldsymbol{H}_{b} \ddot{\boldsymbol{x}}_{b}+\boldsymbol{H}_{b c} \ddot{\boldsymbol{\phi}}+\boldsymbol{c}_{b}=\boldsymbol{w} \boldsymbol{a}+\boldsymbol{z a}, \\
\mathcal{L} & =\boldsymbol{Y} \boldsymbol{a}=\boldsymbol{H}_{b} \dot{\boldsymbol{x}}_{b}+\boldsymbol{H}_{b c} \dot{\boldsymbol{\phi}}=\boldsymbol{W} \boldsymbol{a}+\boldsymbol{Z} \boldsymbol{a},
\end{aligned}
$$

where

$$
\mathcal{F}=\frac{d}{d t} \mathcal{L}, \quad \boldsymbol{y}=\frac{d}{d t} \boldsymbol{Y}, \quad \boldsymbol{w}=\frac{d}{d t} \boldsymbol{W}, \quad \boldsymbol{z}=\frac{d}{d t} \boldsymbol{Z},
$$

and $\boldsymbol{a}$ is a dynamic parameter vector including model inaccuracies. In this paper, we assume that the dynamic model uncertainty appears only in the target, and then the dynamic parameter vector $\boldsymbol{a}$ is defined as a $p$-dimensional vector including the mass, center of mass, moment of inertia and product of inertia of the target:

$\boldsymbol{a}=\left(m, r_{g x}, r_{g y}, r_{g z}, I_{x x}, I_{y y}, I_{z z}, I_{x y}, I_{y z}, I_{z x}\right)^{T},(p=10)$.

$\boldsymbol{Y} \boldsymbol{a}$ and $\boldsymbol{y} \boldsymbol{a}$ represent the linearization forms for the entire system consisting of the chaser-robot and the target. These forms can be separated into two parts. One is related to the motion of the base ( $\boldsymbol{W} \boldsymbol{a}$ and $\boldsymbol{w a}$ ) and the other is for the motion of the active joints $(\boldsymbol{Z} \boldsymbol{a}$ and $\boldsymbol{z a}$ ). The regressor matrices $\boldsymbol{Y}, \boldsymbol{W}, \boldsymbol{Z}, \boldsymbol{y}, \boldsymbol{w}$ and $\boldsymbol{z}$ are functions of the state values and do not include the dynamic parameter uncertainties. Therefore, the errors between the real value and the corresponding expected value are denoted by:

$$
\begin{aligned}
& \widetilde{\mathcal{L}}=\boldsymbol{Y} \overline{\boldsymbol{a}}-\boldsymbol{Y a}=\boldsymbol{Y} \widetilde{\boldsymbol{a}}=\boldsymbol{W} \widetilde{\boldsymbol{a}}+Z \widetilde{\boldsymbol{a}}, \\
& \widetilde{\mathcal{F}}=y \overline{\boldsymbol{a}}-\boldsymbol{y a}=\boldsymbol{y} \widetilde{\boldsymbol{a}}=\boldsymbol{w} \widetilde{\boldsymbol{a}}+\boldsymbol{z} \widetilde{\boldsymbol{a}},
\end{aligned}
$$

where $\widetilde{\boldsymbol{a}}=\overline{\boldsymbol{a}}-\boldsymbol{a}$ denotes a parameter error vector and $\overline{\boldsymbol{a}}$ stands for a vector of estimated parameters.

With assumption $a_{9}$ in Section III, the coupling force between the base-satellite and the robot-arm can be measured. In the case without any external disturbance $(\mathcal{F}=0)$, the measured value is expressed by:

$$
\mathcal{F}_{c}=\boldsymbol{w a}=-\boldsymbol{z a} .
$$

The error between the measured and expected values can be:

$$
\widetilde{\mathcal{F}}_{c}=\boldsymbol{w} \widetilde{\boldsymbol{a}}=-\boldsymbol{z} \widetilde{\boldsymbol{a}} .
$$

Eq. (14) allows us to obtain the parameter error vector $\widetilde{\boldsymbol{a}}$ as:

$$
\widetilde{\boldsymbol{a}}=\boldsymbol{w}^{+} \widetilde{\mathcal{F}}_{c}=-\boldsymbol{z}^{+} \widetilde{\mathcal{F}}_{c},
$$

where the operator $\{\cdot\}^{+}$denotes the right-pseudoinverse. Eq. (15) describes the off-line least-squares estimation technique [9]. The pseudoinverse provides us with the estimated parameter errors that are sensitive to the coupling force errors. Eq. (15) will be used in the derivation of the proposed adaptation method.

\section{B. Coupling Force Based On-Line Parameter Adaptation}

This subsection proposes an on-line parameter adaptation algorithm by considering the momentum characteristic of a free-floating space robot and the coupling force between the base and the robot arm. 
To derive the adaptation algorithm, let us define the following quadratic function as a Lyapunov function candidate:

$$
V(t)=\frac{1}{2}\left(\widetilde{\mathcal{L}}^{T} \boldsymbol{Q} \widetilde{\mathcal{L}}+\widetilde{\boldsymbol{a}}^{T} \boldsymbol{\Gamma} \widetilde{\boldsymbol{a}}\right),
$$

where $\widetilde{\mathcal{L}}$ denotes the momentum error of the entire system, $\boldsymbol{Q} \in R^{6 \times 6}$ and $\boldsymbol{\Gamma} \in R^{p \times p}$ are positive definite matrices, respectively.

Differentiating $V(t)$ yields

$$
\begin{aligned}
\dot{V}(t) & =\widetilde{\mathcal{L}}^{T} \boldsymbol{Q} \widetilde{\mathcal{F}}+\widetilde{\boldsymbol{a}}^{T} \boldsymbol{\Gamma} \dot{\tilde{\boldsymbol{a}}} \\
& =\widetilde{\boldsymbol{a}}^{T}\left\{\boldsymbol{Y}^{T} \boldsymbol{Q}(\boldsymbol{w} \widetilde{\boldsymbol{a}}+\boldsymbol{z} \widetilde{\boldsymbol{a}})+\boldsymbol{\Gamma} \dot{\boldsymbol{a}}\right\} .
\end{aligned}
$$

where $\widetilde{\mathcal{F}}=\frac{d}{d t} \widetilde{\mathcal{L}}$ and eq. (12) are used.

The above equation suggests the choice of the following equality in the sense of the Lyapunov stability theorem:

$$
\boldsymbol{Y}^{T} \boldsymbol{Q}(\boldsymbol{w} \widetilde{\boldsymbol{a}}+\boldsymbol{z} \widetilde{\boldsymbol{a}})+\boldsymbol{\Gamma} \dot{\tilde{a}}=-\boldsymbol{R} \widetilde{\boldsymbol{a}},
$$

where $\boldsymbol{R} \in R^{p \times p}$ is a positive definite matrix. Substituting (14) and (15) into (18), one can obtain the following expression with $\widetilde{\mathcal{F}}_{c}$ :

$$
\boldsymbol{Y}^{T} \boldsymbol{Q}\left(\widetilde{\mathcal{F}}_{c}+\boldsymbol{z} \boldsymbol{w}^{+} \widetilde{\mathcal{F}}_{c}\right)+\boldsymbol{\Gamma} \dot{\tilde{\boldsymbol{a}}}=-\boldsymbol{R} \boldsymbol{w}^{+} \widetilde{\mathcal{F}}_{c} .
$$

That gives us the following adaptation law:

$$
\dot{\tilde{\boldsymbol{a}}}=-\boldsymbol{\Gamma}^{-1}\left\{\boldsymbol{Y}^{T} \boldsymbol{Q}\left(\boldsymbol{E}+\boldsymbol{z} \boldsymbol{w}^{+}\right)+\boldsymbol{R} \boldsymbol{w}^{+}\right\} \widetilde{\mathcal{F}}_{c},
$$

where $\boldsymbol{E} \in R^{6 \times 6}$ denotes the identity matrix.

Accordingly, the time-derivative of $V(t)$ results in:

$$
\dot{V}(t)=-\widetilde{\boldsymbol{a}}^{T} \boldsymbol{R} \widetilde{\boldsymbol{a}} \leq 0 .
$$

The above inequality indicates the proposed adaptation algorithm (20) is asymptotically stable.

\section{Convergence Analysis}

Let us analyze the convergence of the parameters in the proposed algorithm. The proposed adaptation law (20) can be modified to the following expression:

$$
\boldsymbol{\Gamma} \dot{\tilde{a}}+\boldsymbol{R} \widetilde{\boldsymbol{a}}=-\boldsymbol{Y}^{T} \boldsymbol{Q}\left(\boldsymbol{E}+\boldsymbol{z} \boldsymbol{w}^{+}\right) \widetilde{\mathcal{F}}_{c} .
$$

From the expression (22), one can notice that the adaptation law (20) represents a time-varying low-pass filter for $\widetilde{\boldsymbol{a}}$. Therefore, the parameter search in the proposed algorithm (20) goes along a filtered, or averaged, direction.

In addition, the algorithm (20) with the expression (14) leads to the following exponential solution for the parameter error convergence:

$$
\widetilde{\boldsymbol{a}}(t)=\widetilde{\boldsymbol{a}}(0) \exp (\boldsymbol{\beta}),
$$

\begin{tabular}{|c|c|c|c|c|c|c|c|c|}
\hline & mass $[k g]$ & \multicolumn{3}{|c|}{$I_{x x}\left[k g m^{2}\right]$} & \multicolumn{3}{|c|}{$I_{y y}\left[\mathrm{kgm}^{2}\right]$} & $I_{z z}[k$ \\
\hline Base & 140 & \multicolumn{2}{|c|}{18.0} & & \multicolumn{3}{|c|}{20.0} & 22 \\
\hline & & & & \multicolumn{3}{|c|}{ mass $[k g]$} & \multicolumn{2}{|c|}{$I\left[\mathrm{kgm}^{2}\right]$} \\
\hline & \multicolumn{3}{|c|}{ Robot Arm - each Link } & & \multicolumn{2}{|c|}{3.3} & \multicolumn{2}{|r|}{0.0056} \\
\hline & \multicolumn{2}{|c|}{\begin{tabular}{|l|l}
-2 \\
\end{tabular}} & \multicolumn{3}{|c|}{ mass $[k g]$} & \multicolumn{3}{|c|}{$I\left[k g m^{2}\right]$} \\
\hline & \multicolumn{2}{|c|}{ Reaction Wheel } & \multicolumn{3}{|c|}{5} & \multicolumn{3}{|c|}{0.0125} \\
\hline
\end{tabular}

where

$$
\boldsymbol{\beta}=-\int_{0}^{t}\left[\boldsymbol{\Gamma}^{-1}\left\{\boldsymbol{Y}^{T} \boldsymbol{Q} \boldsymbol{y}+\boldsymbol{R}\right\}\right] d t,
$$

in which $\boldsymbol{w}^{+} \boldsymbol{w}=\boldsymbol{E}$ is used. This indicates that the parameter error will converge to zero if

$$
\lim _{t \rightarrow \infty} \int_{0}^{t}\left(\boldsymbol{Y}^{T} \boldsymbol{Q} \boldsymbol{y}+\boldsymbol{R}\right)=\infty .
$$

TABLE I: Dynamic parameters for a chaser-robot

Note that if for all $t \geq 0$,

$$
\int_{t}^{t+T}\left(\boldsymbol{Y}^{T} \boldsymbol{y}\right) \geq \boldsymbol{\alpha},
$$

where $T$ is a time constant and $\boldsymbol{\alpha}$ is a positive constant, the parameter error $\widetilde{\boldsymbol{a}}$ will exponentially converge to zero. In this case, the convergence rate can be found to be $\boldsymbol{\Gamma}^{-1}(\boldsymbol{Q} \boldsymbol{\alpha} / T+$ $R)$.

\section{Simulation STUDY}

This section illustrates the simulation results for the proposed control method. The chaser-robot has a 7-DOF robot arm and three reaction wheels (RW) mounted on the base whose parameters are shown in Table I. The size of the target is the same as that of the chaser-base. In the simulation examples, the control model is assumed to grasp no target while the real chaser-robot grasps the target, giving the extent of uncertainty introduced in the system. For simplicity, the target parameter is included in the dynamic parameters of the end-effector.

Figures 3 and 4 illustrate the angular momentum distribution profiles. Fig. 3 shows the case without parameter adaptation law and Fig. 4 shows the case with parameter adaptation algorithm (20). In the case with adaptation law, the gain $\Gamma^{-1}$ is determined by

$$
\begin{aligned}
\boldsymbol{\Gamma}^{-1}= & \operatorname{diag}([2,0.1,0.1,0.1,1,1,1, \\
& \left.\left.1 \times 10^{-4}, 1 \times 10^{-4}, 1 \times 10^{-4}\right]\right)
\end{aligned}
$$

and the other gains in (20) are set to be identity matrices. In these figures, top graphs and the bottom ones illustrate the momentum profiles of the reaction wheels and those for the base and the robot arm, respectively. In Figs. 3(a) and 4(a), the solid line depicts the total angular momentum of the system, dashed line depicts the accumulated angular momentum onto the reaction wheels. In Figs. 3(b) and 4(b), the solid line and the dashed line depict the angular momentum of the base and that of the robot arm, respectively.

The chaser-robot grasps the target at $t=5[s]$ when one can observe the change of the total angular momentum and the momentum of the base and the robot arm are affected due to the change of the dynamic parameters. After the grasping, the reaction wheels are operated to accumulate the entire angular momentum. In both cases, finally the entire momentum is transfered into the reaction wheels. In the case without parameter adaptation, however, the reaction wheels does not accumulate it properly due to the model inaccuracies while the entire momentum is transfered into the reaction wheels fast in the case with adaptation algorithm. Note here that the 


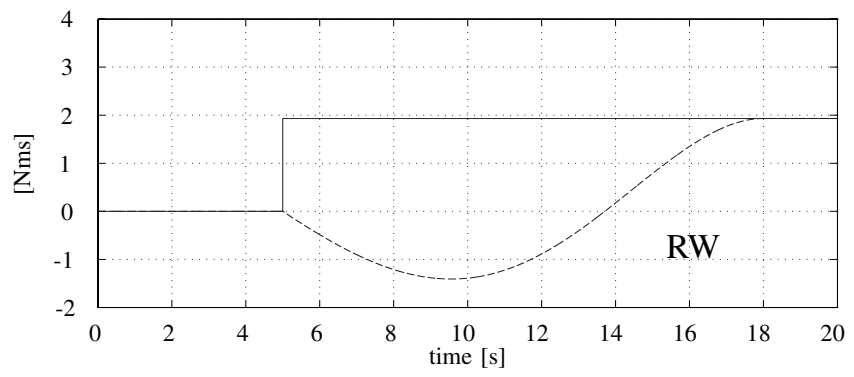

(a) Angular momentum of the reaction wheel $\left({ }^{A} \boldsymbol{H}_{\omega r} \dot{\boldsymbol{\phi}}_{r}\right)$

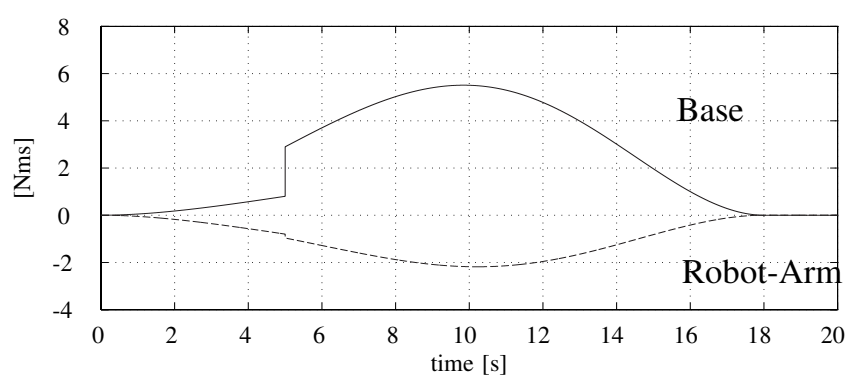

(b) Angular momentum of the base $\left({ }^{A} \boldsymbol{H}_{\omega} \omega_{b}\right)$ and the robot-arm $\left({ }^{A} \boldsymbol{H}_{\omega \phi} \dot{\phi}_{m}\right)$

Fig. 3: Angular Momentum without Parameter Adaptation

performance of the proposed algorithm is limited due to the torque limitation of the reaction wheels in practice.

Fig. 5 illustrates typical examples of the parameter adaptation profile in the case of Fig. 4. The main task here is the prompt momentum accumulation into the reaction wheels and it is not necessary for the dynamic parameters to converge to the real ones. If one needs to identify the real parameters, the persistent-exciting inputs are required.

\section{CONCLUSIONS}

This paper proposed an on-line parameter adaptation to manage the unknown angular momentum for a space robot in the post-grasping of a tumbling target with model uncertainties. Firstly, we discussed a method to accumulate the entire angular momentum into the reaction wheels. The model uncertainties degrade the performance of the proposed momentum accumulation method. To cope with this issue, an on-line adaptation law is proposed by paying attention to the coupling force and the momentum equation. A numerical simulation verified the validity of the proposed adaptation law in the presence of model uncertainties.

\section{REFERENCES}

[1] Y. Xu and T. Kanade, Eds., Space Robotics: Dynamics and Control. Kluwer Academic Publishers, 1993.

[2] Y. L. Gu and Y. Xu, "A Normal Form Augmentation Approach to Adaptive Control of Space Robot Systems," in Proc. of the 1993 IEEE Int. Conf. on Robotics and Automation, vol. 2, Atlanta, USA, May 1993 , pp. $731-737$.

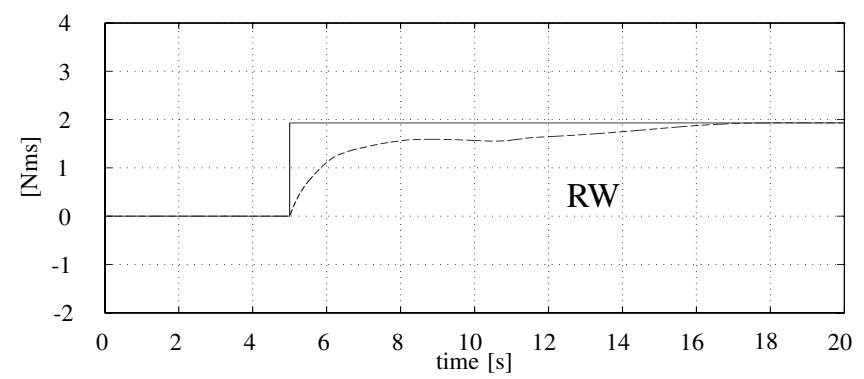

(a) Angular momentum of the reaction wheel $\left({ }^{A} \boldsymbol{H}_{\omega r} \dot{\boldsymbol{\phi}}_{r}\right)$

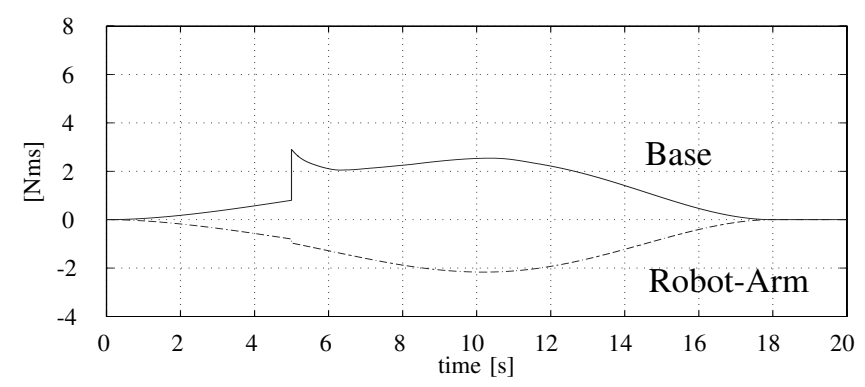

(b) Angular momentum of the base $\left({ }^{A} \boldsymbol{H}_{\omega} \omega_{b}\right)$ and the robot-arm $\left({ }^{A} \boldsymbol{H}_{\omega \phi} \dot{\boldsymbol{\phi}}_{m}\right)$

Fig. 4: Angular Momentum with Parameter Adaptation
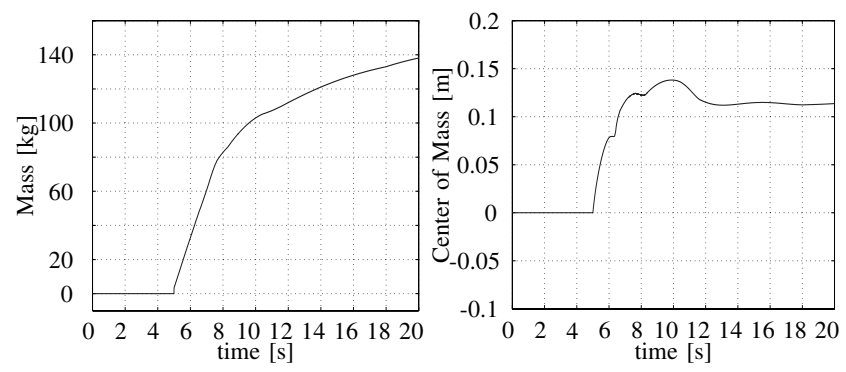

Fig. 5: Parameter Adaptation Profiles

[3] D. N. Dimitrov and K. Yoshida, "Momentum Distribution in a Space Manipulator for Facilitating the Post-Impact Control," in Proc. of the 2004 IEEE/RSJ Int. Conf. on Intelligent Robots and Systems, Sendai, Japan, September. 2004, pp. 3333 - 3338.

[4] D. N. Nenchev and K. Yoshida, "Impact Analysis and Post-Impact Motion Control Issues of a Free-Floating Space Robot Subject to a Force Impulse," IEEE Transactions on Robotics and Automation, vol. 15, no. 3, pp. 548 - 557, June 1999.

[5] K. Yoshida, R. Kurazume, N. Sashida, and Y. Umetani, "Modeling of Collision Dynamics for Space Free-Floating Links with Extended Generalized Inertia Tensor," in Proc. of the 1992 IEEE Int. Conf. on Robotics and Automation, Nice, France, May 1992, pp. 899 - 904.

[6] L.-B. Wee and M. W. Walker, "On the Dynamics of Contact between Space Robots and Configuration Control for Impact Minimization," IEEE Transactions on Robotics and Automation, vol. 9, no. 5, pp. 581 - 591, October 1993.

[7] R. Lampariello, S. Agrawal, and G. Hirzinger, "Optimal Motion Planning for Free-Flying Robots," in Proc. of the 2003 IEEE Int. Conf. on Robotics and Automation, Taipei, Taiwan, May 2003, pp. 3029 3035.

[8] J. J. E. Slotine and W. Li, Eds., Applied Nonlinear Control. Prentice Hall, 1991.

[9] L. Sciavicco and B. Siciliano, Modelling and Control of Robot Manipulators. Springer, 2000. 